\title{
Angular and velocity distributions of tungsten sputtered by low energy argon ions
}

\section{Marenkov, E}

2017-12-01

Marenkov , E , Nordlund , K H , Sorokin , I , Eksaeva , A, Gutorov , K , Jussila , J M A , Granberg , F G \& Borodin , D 2017 , ' Angular and velocity distributions of tungsten

sputtered by low energy argon ions ' , Journal of Nuclear Materials , vol. 496 , pp. 18-23 . https://doi.org/10.1016/j.jn

http://hdl.handle.net/10138/307105

https://doi.org/10.1016/j.jnucmat.2017.09.021

cc_by_nc_nd

acceptedVersion

Downloaded from Helda, University of Helsinki institutional repository.

This is an electronic reprint of the original article.

This reprint may differ from the original in pagination and typographic detail.

Please cite the original version. 


\title{
Angular and velocity distributions of tungsten sputtered by low energy argon ions
}

E. Marenkov ${ }^{1}$, K. Nordlund ${ }^{1,2}$, I. Sorokin ${ }^{1,4}$, A. Eksaeva ${ }^{1,3}$, K. Gutorov ${ }^{1}$, J. Jussila ${ }^{2}$, F. Granberg $^{2}$, D. Borodin ${ }^{3}$

${ }^{1}$ National Research Nuclear University MEPhI (Moscow Engineering Physics Institute), 115409, Moscow, Kashirskoe shosse, 31

${ }^{2}$ Department of Physics, P.O. Box 43, FI-00014, University of Helsinki, Finland.

${ }^{3}$ Forschungszentrum Juelich GmbH, Institut fuer Energie- und Klimaforschung - Plasmaphysik, 52425 Juelich, Germany

${ }^{4}$ Kotel'nikov Institute of Radio Engineering and Electronics, Fryazino Branch,

Russian Academy of Sciences, pl. Vvedenskogo 1, Fryazino, Moscow oblast, 141190 Russia

Corresponding author: Evgeny Marenkov, edmarenkov@gmail.com, National Research Nuclear University MEPhI (Moscow Engineering Physics Institute), 115409, Moscow, Kashirskoe shosse, 31

\begin{abstract}
Sputtering by ions with low near-threshold energies is investigated. Experiments and simulations are conducted for tungsten sputtering by low-energy, $85-200 \mathrm{eV}$ Ar atoms. The angular distributions of sputtered particles are measured. A new method for molecular dynamics simulation of sputtering taking into account random crystallographic surface orientation is developed, and applied for the case under consideration. The simulations approximate experimental results well. At low energies the distributions acquire "butterfly-like" shape with lower sputtering yields for close to normal angles comparing to the cosine distribution. The energy distributions of sputtered particles were simulated. The Thompson distribution remains valid down to near-threshold $85 \mathrm{eV}$ case.
\end{abstract}

\section{Highlights}

Angular and energy distributions of sputtered particles are considered for low-energy sputtering. A new algorithm for molecular dynamics simulations of polycrystalline target is proposed. Results of the simulations agree with experimental findings both obtained by us and found in literature.

\section{Introduction}

Interaction of plasma with the first wall and divertor materials has been recognized as one of the major problems for using tokamaks as future fusion power plants. Steady-state erosion of the plasma-contacting materials and even larger damage caused by non-steady state events such as disruptions, giant ELM (edge localized mode) bursts, MARFE (multifaceted asymmetric radiation from the edge), and others, can lead to severe consequences, ranging from discharge 
termination to heavy wall damage [1]. These issues can jeopardize successful implementation of a tokamak type reactor for long-term operation and energy production.

The main mechanism of the tungsten first wall steady-state erosion is physical sputtering by plasma ions having low energy $<100 \mathrm{eV}$, but very high fluxes, of the order of $10^{20}$ $\mathrm{cm}^{-2} \mathrm{~s}^{-1}$. During many years of physical sputtering research, a good overall understanding of the process has been achieved. Depending on the projectile energy, three regimes can be distinguished. At moderate energies, typically $1-10 \mathrm{keV}$, the projectile can transfer enough energy to a target atom which have enough momentum to displace another target atom, forming a linear cascade of collisions. At higher energies, the density of displaced atoms is so high that many-body collisions between them cannot be neglected, leading to a region of a dense very hot liquid-like zone, known as the thermal spike [2,3]. And finally, at the very low energies well below $1 \mathrm{keV}$, sputtering occurs in the "single knock-off" regime, when a cascade cannot appear and a surface atom is kicked off from its position either directly by the projectile itself, or after several collisions taking place in the topmost surface layers.

The linear cascade regime is described by kinetic equations giving the distribution function of the target atom displacements [4]. In general, this approximation gives excellent agreement with experimental results both for sputtering yield values, as well as for the angular and energy distributions of sputtered particles. The differential sputtering yield $(Y)$ for sputtered atoms with energy $E$ flying within solid angle $d \Omega=2 \pi \sin \theta \mathrm{d} \theta$ around the surface normal is expressed as [5]

$$
\frac{\partial^{2} Y}{\partial E \partial \Omega}=C_{m} \frac{E}{(E+U)^{3-2 m}} \cos \theta
$$

where $C_{m}$ is the normalization constant, $U$ is the surface binding energy, and $m$ is the fitting parameter. This relation, however, holds only for a certain energy range depending on target material and projectile.

At lower energies, this "kinetic" approach does not work. The sputtering occurs in the few-collisions regime. As a result angular distribution of sputtered atoms is not cosine, but has a "butterfly-like" shape [6]. The energy distribution [7] does not change very significantly, but the maximum position depends on energy of projectiles and therefore is not defined just by the surface binding energy $U$.

It seems that the only way to obtain analytical results in this case is to carefully consider all possible collision sequences occurring in the first surface layers and leading to sputtering. This was done in $[8,6]$. However, no analytical expressions were obtained for energy or angular distributions of sputtered particles. Instead the authors focused on derivation of the threshold 
energies for sputtering in different cases. We also notice that there are several assumptions involved in the consideration: atoms are supposed to move only in one plane, although sputtering is essentially a 3D proves; only binary collisions were taken into account, which is not the case for low energy sputtering. Therefore it looks like the more reliable way for sputtering research at these energies is computer simulation.

SRIM-like codes based on binary-collision approximation (BCA) are very often used for simulation of sputtering [9]. However, the approach has principal drawbacks for low energies. First, at low energies many-body collisions start to play a significant role. Therefore, it is impossible to account for some experimentally observed phenomena, for example sputtering of clusters [10]. Second, the SRIM Monte Carlo algorithm for the selection of the next colliding ion works as if the target has an amorphous structure. This is a good approximation for high energy sputtering, when long collision cascades are developed. In the few collisions regime, the collision sequence strongly depends on individual atom positions, implying that the full crystal structure has to be modeled. One of experimental evidences for the role of crystal structure is an anisotropic peak shape of angular distributions of sputtered atoms often referenced to as "Wehner-spots" [10].

Much better results can be obtained using molecular dynamics (MD) simulation technique [11]. Many-body potentials are routinely employed for the calculations, and these also include the attractive interaction between atoms, thus inherently giving a description of the surface binding energy of atoms which is a free parameter in binary-collisions codes. The crystal lattice structure is also accounted for by default.

The few-collisions regime is important for steady-state erosion of first-wall materials in tokamaks as it provides a constant, source of plasma impurities. Experiments performed at linear-plasma devices [12] have shown the importance of angular and energy distributions of sputtered particles for understanding its transport in the fusion plasma. At the same time, there is very few data available in literature for the distributions at low energies.

Although sputtering by hydrogen isotopes is the most interesting for fusion research many experimental works are done with heavier ions, e.g. argon (Ar) or neon [12]. This is because sputtering yields for deuterium sputtering are very low which causes additional experimental difficulties. On the other hand physical sputtering mechanisms do not depend on the projectile type. Numerical simulations using MD provide a necessary link for extrapolating the results to ions of interest.

In this work, we present experimental results on tungsten (W) sputtering by argon (Ar) for low energies $85-200 \mathrm{eV}$. The angular distributions of sputtered particles are measured by deposition on witness plates. Experimental results are compared with simulations by TRIM-like 
code SRIM and the molecular dynamics code PARCAS. Molecular dynamic simulations are done using a new technique accounting for contribution of different random crystal faces, allowing for a better imitation of polycrystalline sample structure.

\section{Experimental results}

Experiments on tungsten sputtering by argon ions were done at PR-2 facility [13]. PR-2 is a magnetic mirror linear plasma trap using the beam-plasma discharge. A polycrystalline tungsten sample was situated on the watercooled electrical feedthrough in the face of the plasma column, fig. 1. The usual diameter of the plasma column (about $3-6 \mathrm{~cm}$ ) was limited by a diaphragm at the magnetic mirror. The area of the plasma spot at the centre of the sample was $0.75 \mathrm{~cm}^{2}$. The energy of the sputtering ions was controlled by applying biasing voltage to the sample. The energy spread is defined mainly by the plasma potential ( 20 V). Parameters of the plasma were fixed for all the experiments: the pressure - $0.1 \mathrm{~Pa}$, power of the electron gun - 220 $\mathrm{W}(1.3 \mathrm{kV} \times 170 \mathrm{~mA})$, ion flux to the $\mathrm{W}$ sample $-5 \times 10^{16} \mathrm{~cm}^{-2} \mathrm{~s}^{-1}$. The plasma parameters were monitored by a single Langmuir probe and by magnetic mass-spectrometer measurement of the mass-spectrum of the ion flux [14]. The collecting system consisting of four $\mathrm{Cu}$ ribbons bent with $7.6 \mathrm{~cm}$ radius segments was mounted around the sample, see fig. 1. The experiments were carried out for low energy of sputtering ions 100, 150 and $200 \mathrm{eV}$ with fixed exposure time 23 hours. The exposure time has been chosen so that there is enough $\mathrm{W}$ deposited on all the collectors.

To determine the angular distributions, we analyzed the thickness of the tungsten layers deposited on the copper ribbons. The thickness of the deposited $\mathrm{W}$ film has been determined by energy-dispersive X-ray spectroscopy (EDS) $[15,16]$. The ratio of intensity of the characteristic X-ray emission lines of the film (W) and substrate material $(\mathrm{Cu})$ was obtained. The ratio depends on the film thickness. Monte-Carlo simulations of X-ray emission were then conducted using CASINO code [17]. The calculations were carried out with fixed incident electron energy $5 \mathrm{keV}$, corresponding to SEM setup of the electron injector during the analysis, and number of the electrons to simulate $10^{4}$. The statistical accuracy of the MC simulation was about $1 \%$. Comparing the results of simulations to the measured X-ray intensities it is possible to obtain the film thickness.

Measurements of the $\mathrm{W}$ thickness have been taken in 18 points along the each $\mathrm{Cu}$ plate corresponding to angle ranges of $15-90^{\circ}$. The angle range was limited by the plasma column diameter at small angles and the $\mathrm{W}$ sample fixture at large angles. The film thickness for the each sputtering angle was calculated as average value over $4 \mathrm{Cu}$ ribbons. The accuracy of the 
method is limited by the inhomogeneity of the W film within the EDS spot and accuracy of Monte-Carlo simulations of X-ray measurements, and is estimated to be about $1 \mathrm{~nm}$. Since EDS collects the signal from the same area for all the samples, the $\mathrm{W}$ film thickness is proportional to differential sputtering yield $d Y / d \Omega$. However, in the figures below we plot $d Y / d \theta=2 \pi \sin \theta d Y / d \Omega$ distributions because they are naturally obtained in our calculations. Experimental angular distributions normalized by the maximum film thickness are shown in fig. 2. The error bars are plotted as normalized experimental error $(1 \mathrm{~nm})$ by maximum value of the $\mathrm{W}$ thickness.

We see that for all three energies, the angular distributions are well approximated by the cosine distribution at large angles. At small angles the deviation from the cosine law is notable. All the experimental data shows lower sputtering for $\theta<45^{\circ}$ than is expected. The maximum position according to the results of SRIM simulations (ver: SRIM-2008.04., [18]) is shifted towards the lower angles confirming invalidity of binary-collisions approximation for low energies. MD simulations by PARCAS code also plotted in fig. 2 are discussed below.

The total sputtering yield also can be estimated from EDS thickness measurements. The total number of sputtered atoms is defined by the film thickness. This value has to be extrapolated on the hemisphere because the collector ribbons cover only small part of the sphere, and also because sputtered particles flying too close to the PR-2 axis remain unregistered. This is done assuming uniform distribution of sputtered particles around the axis and cosine law at small angles. The extrapolation and the film thickness measurements are the main sources of the errors of the method. The obtained sputtering yields are plotted in fig. 3 together with data from [5]. There is a fairly good agreement between both results. Simulations performed with a SRIM code give the same dependence of the sputtering yield on the energy, although the magnitude is systematically larger than the experimental data.

\section{Molecular dynamics simulations}

As it was noted in Introduction binary collision codes cannot be trusted for low energy sputtering simulations, as the approximation of binary collisions is violated. Molecular dynamics presents a more reliable tool for modeling in this energy range. The typical procedure used in MD simulation includes preparation of the "sample" and bombardment itself [19]. The principle drawback of the MD simulations is that usually only one or several crystal planes are considered. One notices, however, that the experimental samples are polycrystalline as well as the first wall materials. Lattice planes with arbitrary Miller indexes may be present at the surface, depending on materials manufacturing and sample preparation procedure. Molecular dynamic simulations have to be done for all the plane orientations to account for this effect. The average then should 
be taken to obtain the realistic angular and energy distributions. Results of such calculations are different from TRIM-like simulation of amorphous targets. Even though many planes with different orientations are mixed together, development of a few-collisions cascade within one plane orientation differs from collision pattern in fully amorphous target [20].

We implement an algorithm to simulate random surface orientation in MD in the following way. We first created a cube with side length $r_{s}+10 \AA$, and rotated this randomly with 3 randomly selected Euler angles $\alpha, \beta, \gamma$, out of which the $\beta$ angle was selected weighted by $\cos ^{-1}(2 u)$ to weight solid angles correctly in $3 \mathrm{D}$ ( $u$ is a random number between 0 and 1$)$. The cube was created out of $\mathrm{W}$ in the body-centered cubic structure, with a lattice constant corresponding to $300 \mathrm{~K}$ in the interatomic potential chosen. From the rotated cube, a hemisphere of atoms of radius $r_{s}$ was cut out. The sphere center coincides with the cube center. The hemisphere was simulated using 3 radial layers: (A), (B) and (C), fig. 4. The innermost region (A) up to $r_{s}-10 \AA$ was simulated in the NVE ensemble, conserving number of particles, volume and energy, to handle the many-body collisions created by the incoming ion correctly. In the region (B) $\left[r_{s}-10, r_{s}-3\right] \AA$ A the temperature of the atoms was scaled with Berendsen method [21] to absorb the extra heat introduced to the system by the kinetic energy of the incoming projectile. Finally, atoms in the region (C) $\left[r_{s}-3, r_{s}\right] \AA$ were held fixed to prevent cell motion. After creation of the randomly rotated cell, it was first simulated once at $300 \mathrm{~K}$ for $10 \mathrm{ps}$ without any ion bombardment to relax the surface.

After this, Ar bombardment was conducted on the relaxed plane surface in region (A). An Ar ion is initially positioned $5 \AA$ above the hemisphere plane surface in a random place selected over a size of $4 \times 4$ lattice constants, and directed perpendicularly towards the surface with the desired energy. For each randomly generated surface, 5000 incoming ions were simulated, and for each energy, this procedure was repeated over at least 19 different randomly oriented surfaces.

The results of calculations for 100, 150 and $200 \mathrm{eV}$ are shown in fig. 2. One sees that our method of simulations gives good agreement between MD and experimental findings. Even the depletion of the low-angular part of the distribution is reproduced well.

The sputtering yields were also defined from the simulations and are plotted in fig. 3 . The MD values are systematically larger than experimental results, but have the similar slope.

Next we simulate $85 \mathrm{eV}$ Ar sputtering. The corresponding angular distribution can be found in [22] where it was measured using a quadruple mass spectrometer. Results of the simulations are shown in Fig. 5 along with experimental data, SRIM results and cosine 
distribution. As no absolute sputtering yield value was determined in [22], the presented curve is normalized to fit our simulations by magnitude. To not overload the picture, only distribution averaged over different surface orientations is shown. Notice that SRIM simulations give totally different distribution, which has a more pronounced maximum shifted towards smaller angles. A feature of the experimental distribution is a maximum at approximately $80^{\circ}$. The MD calculations do not reproduce the maximum perfectly, but demonstrate the similar trend. The obtained distribution has fewer atoms sputtered in small angles comparing to the cosine distribution and has additional atoms within larger angles. This last feature resembles the experimental maximum. Thus one can say that at smaller projectile energies the number of particles sputtered close to the surface normal is reduced comparing to the cosine distribution. Eventually the distribution becomes "butterfly-like" [6]. However, this shape establishes well only at very low energies close to the sputtering threshold.

The distributions obtained from different random surface orientations are shown in Fig.6(a, b) for $200 \mathrm{eV}$ and for $85 \mathrm{eV}$. At larger energy collisions go deeper into the surface reducing the difference between distributions from various plain orientations.

To understand the sputtering physics for $85 \mathrm{eV}$ case in more details we have analyzed the trajectories of the sputtered $\mathrm{W}$ atoms as well as those in the cell region within $1 \mathrm{~nm}$ of the Ar impact point, in cases where sputtering did occur. Visual analysis of more than 10 individual cases showed that indeed at this low energy, sputtering of $\mathrm{W}$ occurs almost only when an $\mathrm{Ar}$ atom hits a $\mathrm{W}$ atom in the top layer from the side. Two examples are shown in fig. 7 for the $<001>$ plane and $85 \mathrm{eV}$ sputtering. First of all, the sputtering processes are localized within the several topmost layers, implying the strong influence of surface condition on the experimental results. Surface contamination with oxides or any other impurities as well as defects in the surface structure can influence the collision sequence significantly. Secondly, one can see that all the surrounding atoms move, showing that the sputtering in this case indeed cannot be described accurately in binary collisions approximation.

In principle, two mechanisms are possible in few-collision sputtering. The first one is for an Ar atom to hit $\mathrm{W}$ atom, which is then scattered by one of the surrounding atoms and flies away. For larger energies this process initiates a collision cascade. The second one is that $\mathrm{Ar}$ atom is scattered by one of the $\mathrm{W}$ atoms and then kicks out a $\mathrm{W}$ atom from the topmost layer. It is possible to estimate threshold energies for sputtering in both cases from binary collisions kinematics. The corresponding calculations were done in [8]. Using eq. (14) from that paper we find that the threshold energy for sputtering by the first mechanism is $16 U / \gamma \approx 220 \mathrm{eV}$, where $\gamma=4 M_{1} M_{2} /\left(M_{1}+M_{2}\right)^{2}$ is the energy transmission factor, $M_{1,2}$ are masses of the target and 
projectile atoms. It means that long collision cascades cannot develop for lower energies, thus giving the lower estimate for application of classical linear cascade theory. The sputtering threshold for the second mechanism is given by eq. (47)-(48) from the same work [8]. The energy in our case is about $20 \mathrm{eV}$. This energy is only slightly larger than estimate for the lowest possible threshold $E_{t h}=U_{s} / \gamma \approx 14 \mathrm{eV}$. This is not surprising however since the mass ratio of $\mathrm{Ar} / \mathrm{W}$ is about 0.2, meaning that an Ar atom can be scattered almost without losing the energy from heavier target atoms and finally sputter W. The <001> surface therefore gives a good opportunity for such sputtering, as there are $\mathrm{W}$ atoms in the second layer which can be hit directly by Ar passing through the first layer without collision (see fig. 7). This process is impossible for other crystal orientations, e.g. 011, where the first layer is closely packed and the second layer is not directly seen by Ar. However, more closed-packed planes are also easier to sputter at larger energies due to increased probability for projectile to hit a target atom.

From fig. 7 we see that the second mechanism is indeed important in our case. Notice also that the short collision sequence suggests existence of a well defined most probable angle of sputtered atom emission. That is the reason for the maximum at $80^{\circ}$ appearing at both experimental and simulated distributions.

Another valuable question for understanding of plasma impurity transport is the energy distribution of sputtered particles. The distributions obtained by MD "averaged planes" method are shown in Fig. 8 for different projectile energies. The dashed curves are the best fits by Thompson distribution (1.1)200 eV case is well described by classical cascade theory $m=0$ [o- $m$ $U=8 \mathrm{eV} 23]$. Maximum is located at $E_{0}=U / 2(1-m)$ The $150 \mathrm{eV}$ distribution is fitted with the same parameters. However, $85 \mathrm{eV}$ case cannot be well approximated by Thompson function even if one varies $m$ obs $m$ independently. The tail of the simulated distribution decreases more rapidly than Thompson distribution suggests.

The difference is expected since the linear cascade theory is not applicable for such low energies. Some authors report that there is a slight shift in maximum position for few-collision case towards smaller energies [7]. Our simulations do not reproduce this effect.

\section{Conclusions}

The angular and energy distributions of sputtered particles for low energy sputtering were investigated using $\mathrm{W}$ sputtering by $\mathrm{Ar}$ at $85-200 \mathrm{eV}$ range as an example. The experiments were conducted at PR-2 facility using witness plates for measurements of angular distributions. The experimental results show that the cosine distribution describes generally well the angular distribution shape down to low energies, $>100 \mathrm{eV}$, at least for not too small angles. 
This may seem surprising, as cosine distributions are typically associated with linear cascade sputtering which is applicable for higher energies, $>1 \mathrm{keV}$.

A new methodology for molecular dynamics simulations was applied to simulate the corresponding processes. The method allows considering surface planes with different random orientations, therefore imitating polycrystalline structure of real materials. The simulations reproduce well experimental results, namely smaller sputtering close to the surface normal than given by the cosine distribution.

The sputtering of lower energies occurring in few-collisions approximations was also simulated for $85 \mathrm{eV}$ Ar projectiles. Results of the simulations have the same trend as experimental findings in [22]. The role of crystal geometrical structure is important for fewcollision sputtering. It leads to pronounced difference in angular distributions of atoms sputtered from different planes. The real distribution averaged from different planes orientations differs from the cosine. The reason for the difference is preferential sputtering directions becoming important in few-collision mechanism.

Regarding the energy distributions we found that they can be well described by Thompson formula when the angular distribution is approximately cosine. At very low energies, $85 \mathrm{eV}$ for $\mathrm{Ar}-\mathrm{W}$ sputtering, the Thompson distribution does not fit the results of the simulations, although we do not notice any sufficient shift of the distribution maximum reported in other works [7].

\section{Acknowledgments}

The work is supported by Russian President grant 14.Y30.17.3098-MK 


\section{References}

[1] G. Arnoux, A .Loarte, V. Riccard1, W. Fundamenski, A. Huber., Nucl. Fusion 49 (2009) 085038

[2] J. Samela and J. Kotakoski and K. Nordlund and J. Keinonen, Nucl. Instr. Meth. Phys. Res. B. 239, 331-346 (2005)

[3] K. Nordlund and F. Djurabekova, J. Comput. Electr. 13, 122 (2014)

[4] P. Sigmund, Physical Reviews. 184, 2 (1960)

[5] R. Behrisch, W. Eckstein. Sputtering by Particle Bombardment. Springer-Verlag Berlin Heidelberg 2007

[ 6] Y. Yamamura, Y. Mizuno, H. Kimura, Nuclear Instruments and Methods in Physics Research B13, 393-395 (1986)

[7] R. A. Brizzolara, C. B. Cooper, T. K. Olson, Nuclear Instruments and Methods in Physics Research B35, 36-42 (1988)

[8] Y. Yamamura, J. Bohdansky, Vacuum 35, 12 (1985)

[9] H. Hofsäss, K. Zhang, A. Mutzke, Applied Surface Science 310, 134-141 (2014)

[10] G. Betz, K. Wien. International Journal of Mass Spectrometry and Ion Processes 140, 1-110 (1994)

[11] H. M. Urbassek, Nuclear Instruments and Methods in Physics Research B 122, 427-441 (1997)

[12] E. Marenkov, A. Eksaeva, D. Borodin et al., Journal of Nuclear Materials 463, 268-271 (2015)

[13] Gutorov K.M., J. Surf. Investig. 10, 612-616 (2016)

[14] I. Sorokin, I. Vizgalov, D. Kolodko. AIP Conference Proceedings 1771, 050010 (2016)

[15] N. Pryds et al., Appl. Surf. Sci. 252, 4882-4885 (2006)

[16] H.E. Bishop, D.M. Poole , J. Phys. D. Appl. Phys. 6, 1142-1158 (1973)

[17] H. Demers et al., Scanning, 33, 135-146 (2011)

[18] http:// www.SRIM.org

[19] M. Ghaly, K. Nordlund, R. S. Averback, Phil. Mag. A 79, 795 (1999)

[20] K. Nordlund, F. Djurabekova, and G. Hobler, Phys. Rev. B 94, 214109 (2016)

[21] H. J. C. Berendsen, J. P. M. Postma, W. F. van Gunsteren, A. DiNola, J. R. Haak, J. Chem. Phys., 81(8):3684, (1984) 
[22] D. Nishijima, M.J. Baldwin, R.P. Doerner, J.H. Yu, Journal of Nuclear Materials, 415, S96S99 (2011)

[23] R. Szwarc, E. R. Plante, J. J. Diamond, Journal of research of the Notional Bureau of Standards - A. Physics and Chemistry, 69A (1965) 


\section{Figure captions}

Fig. 1. A scatch of the assembly designed for mesuarments of the angular distributions.

Fig. 2. Angular distributions mesuared at PR-2 (line with circles) ploted together with cosine distribution (dashed line) and SRIM calculations (line with squares). The PARCAS simulation results are marked by open triangles.

Fig. 3. The sputtering yields versus the projectile energy. SRIM and PARCAS results are compared with experimental data from Behrisch [5] and our results (the line with triangles).

Fig. 4. The molecular dynamic simulations setup. The innermost (A) and outermost (C) regions are shown in blue, the intermediate region (B) is shown in red.

Fig. 5. Angular distribution for $85 \mathrm{eV}$ bombardment. Experimental results from Nishijima [22] paper are better approximated by PARCAS simulations averaged over 20 random surface planes orientations then by cosine distribution (dashed curve) or by the SRIM code.

Fig. 6. Angular distiburion for $200 \mathrm{eV}$ (a) and $85 \mathrm{eV}$ (b) bombardment calculated with the PARCAS code. The dashed line is cosine distribution and the broken lines are distribution from surface planes with random orientations.

Fig. 7. Typical sputtering events for low energy sputtering, $85 \mathrm{eV}$. Surface orientation is $\langle 100\rangle$.

Fig. 8. The energy distributions of sputtered particles for different projectile energies simulated with PARCAS. 


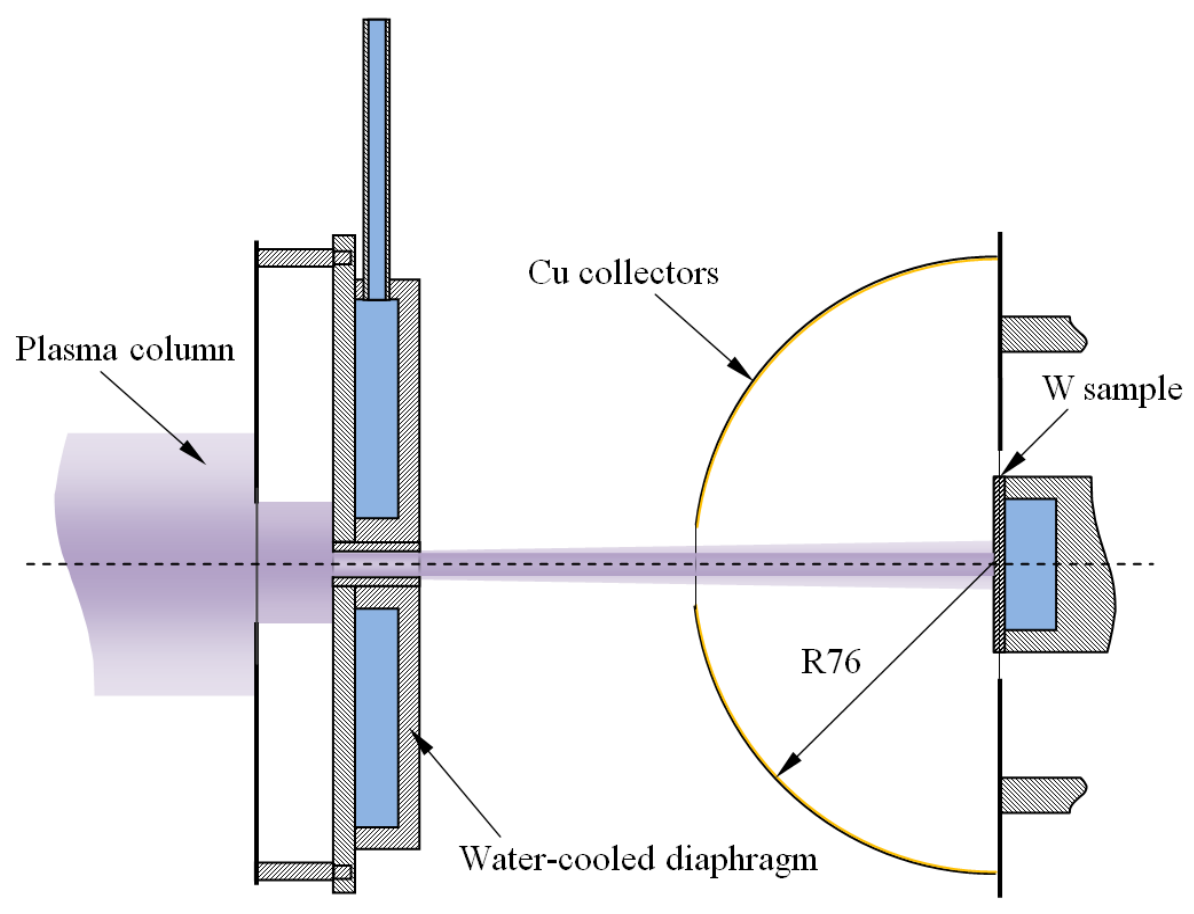

Fig. 1

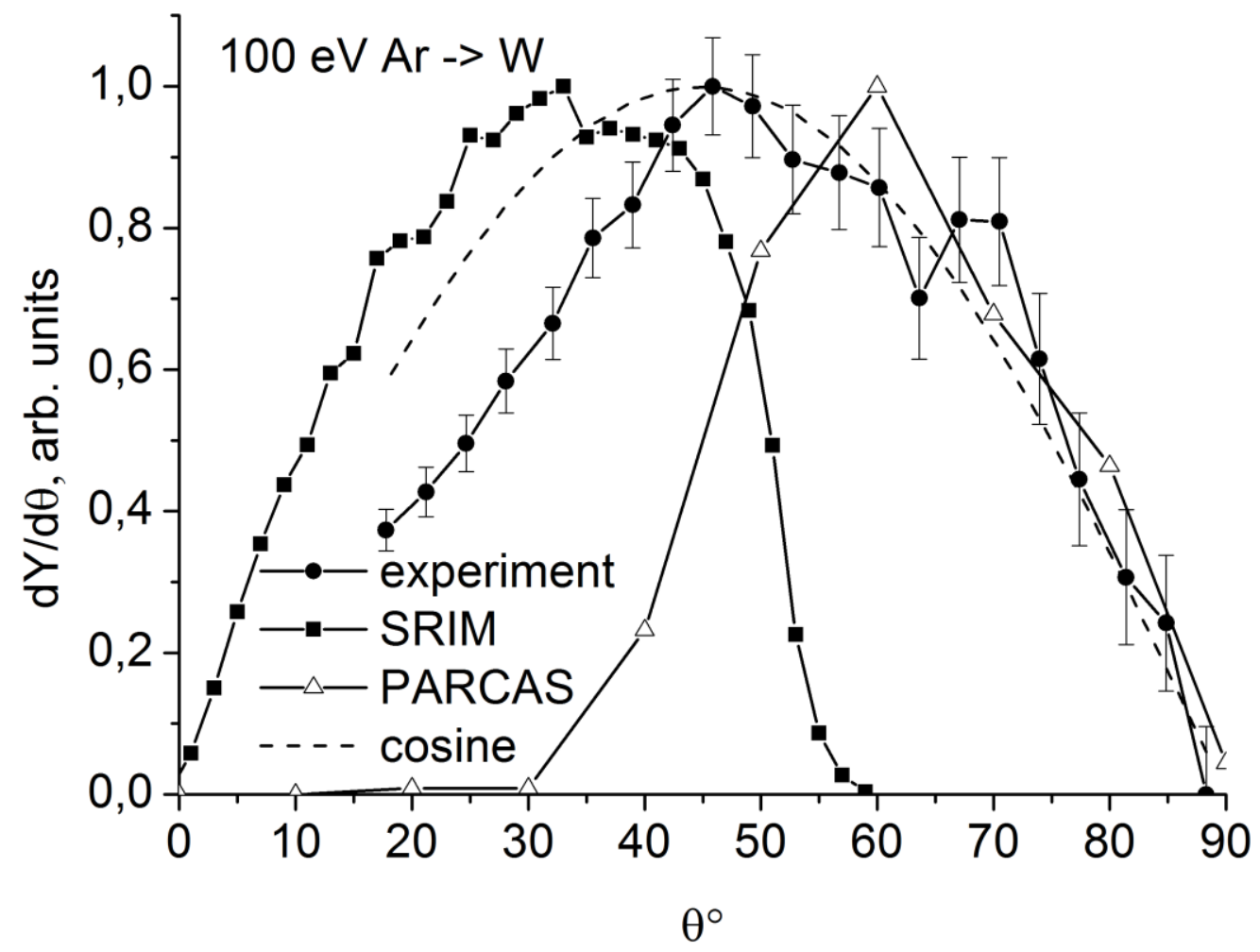

Fig. 2a 


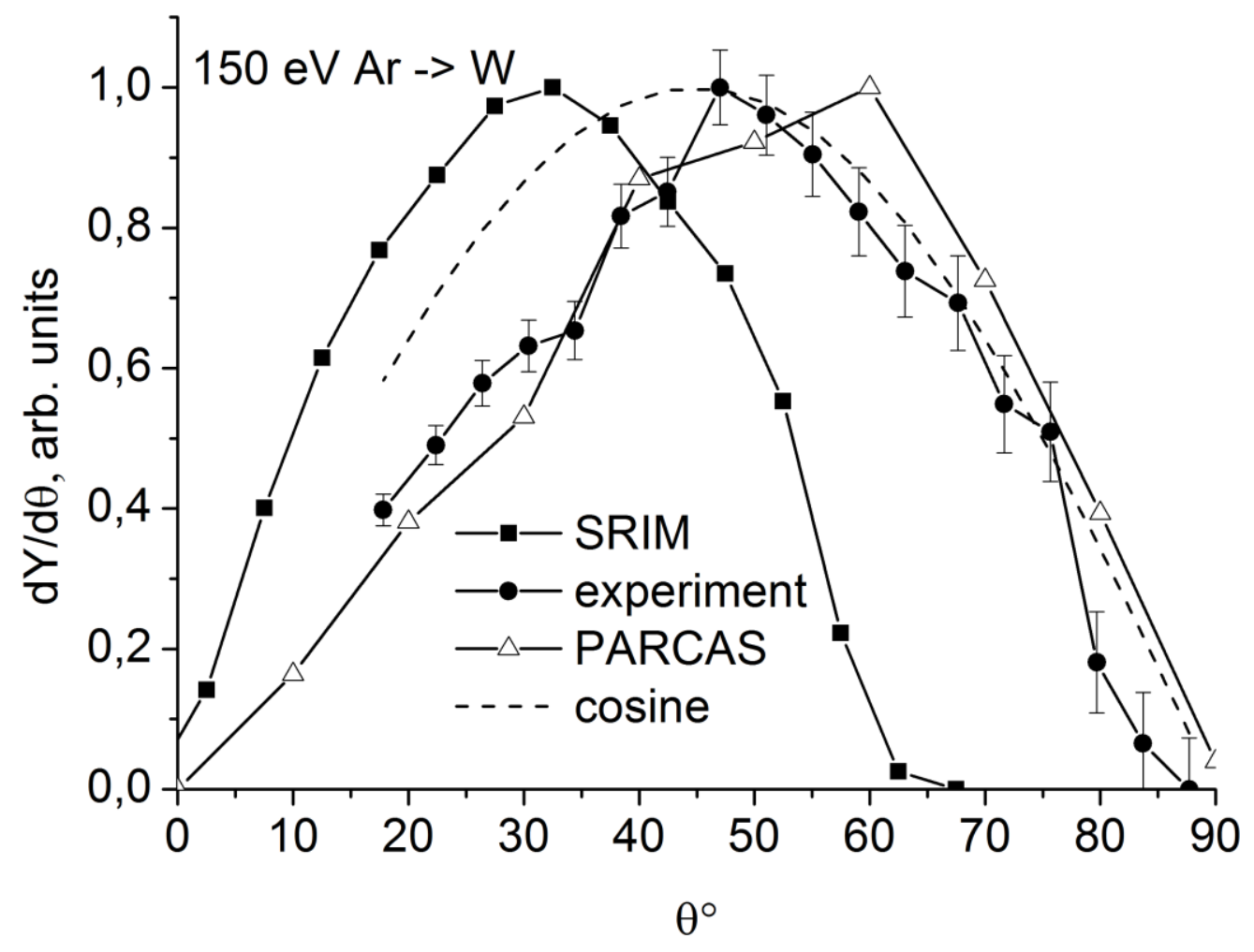

Fig. $2 b$

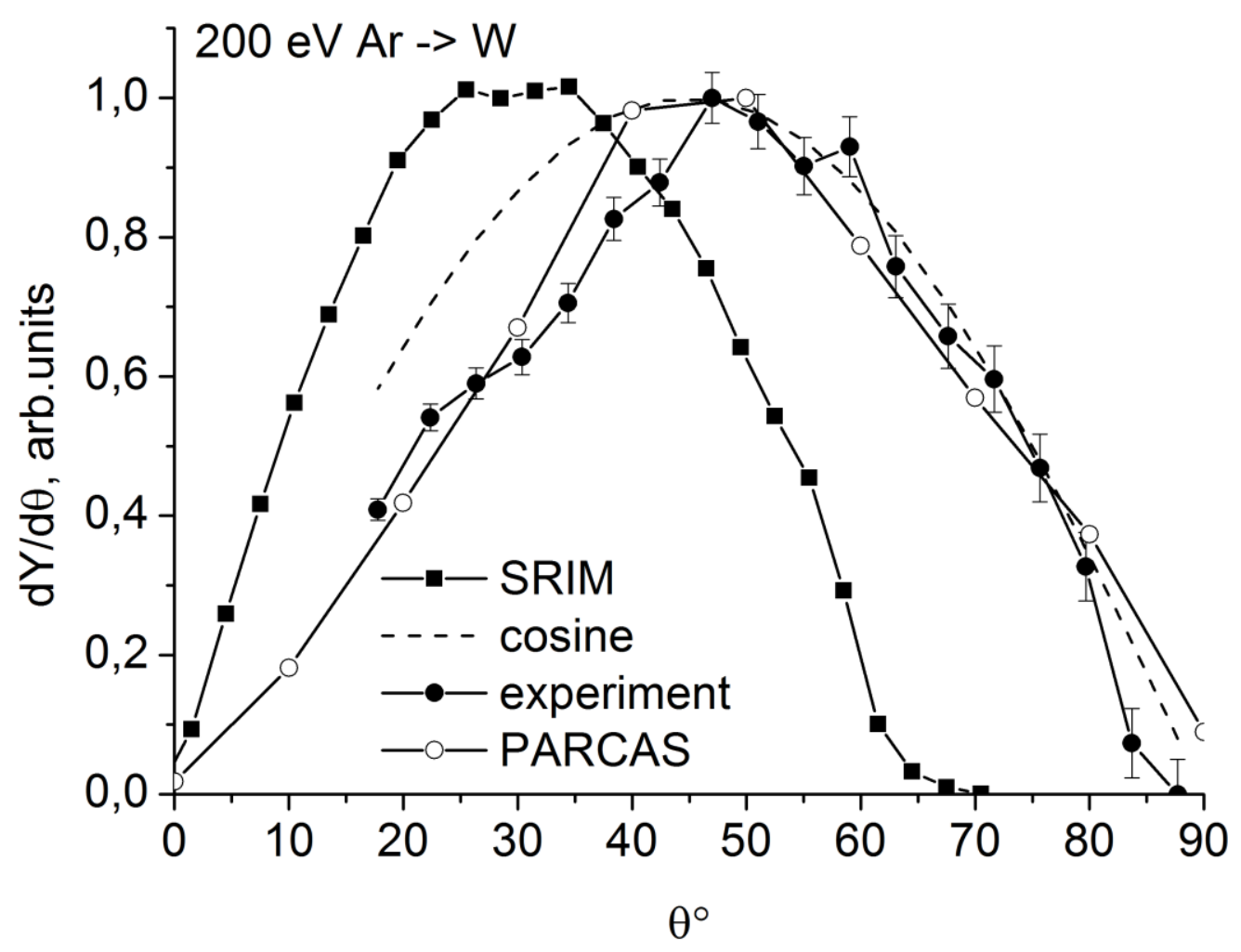

Fig. 2c 


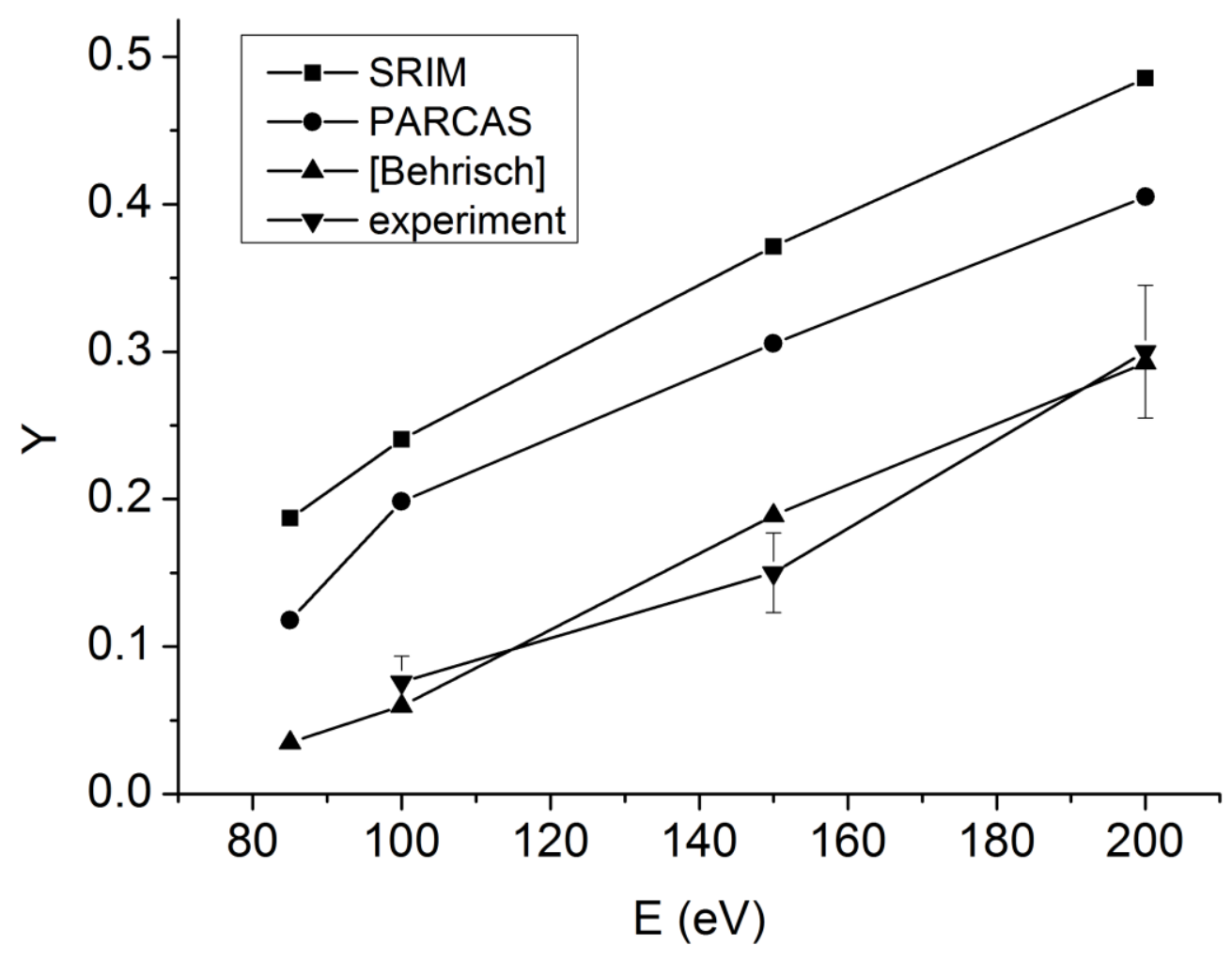

Fig. 3

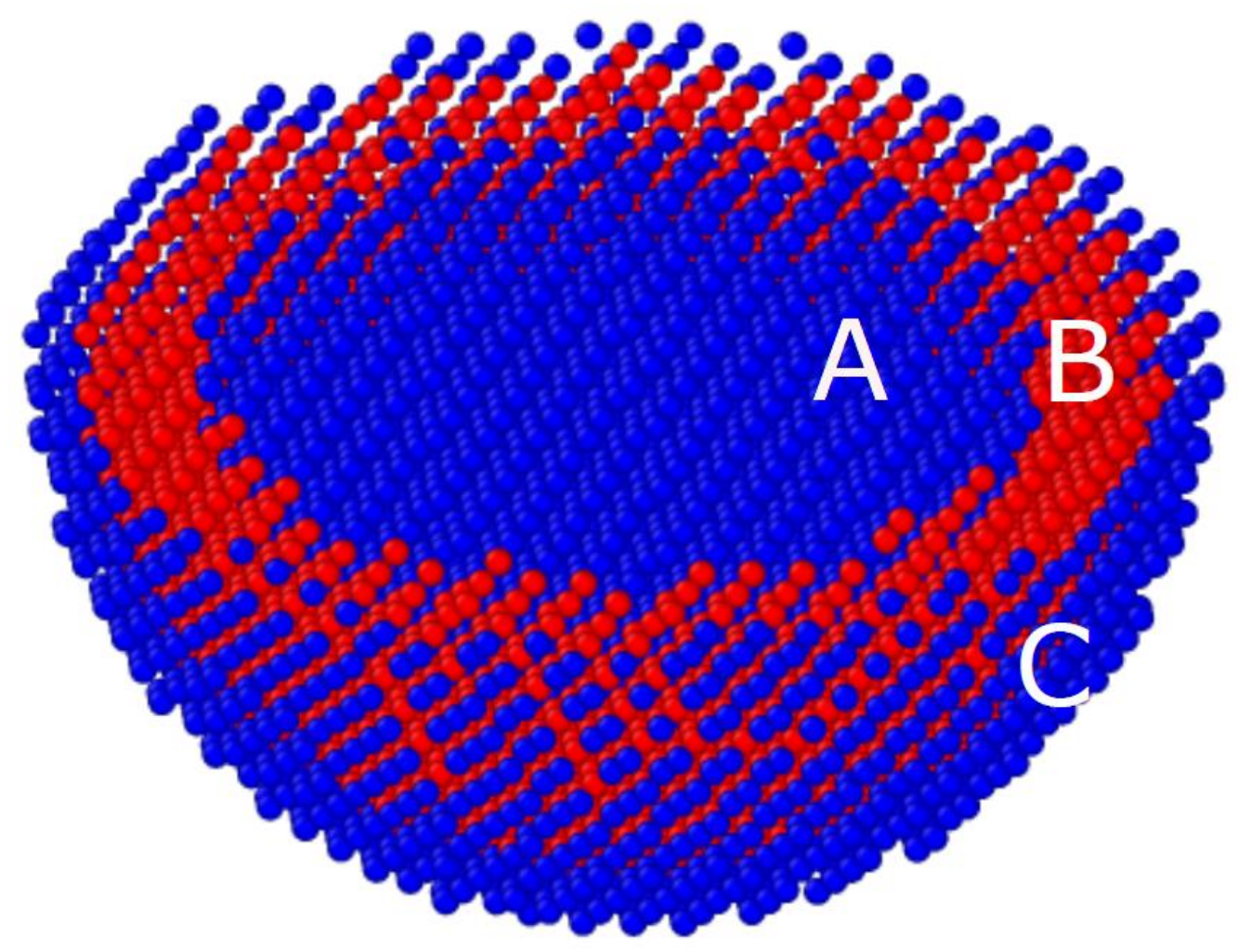

Fig. 4 


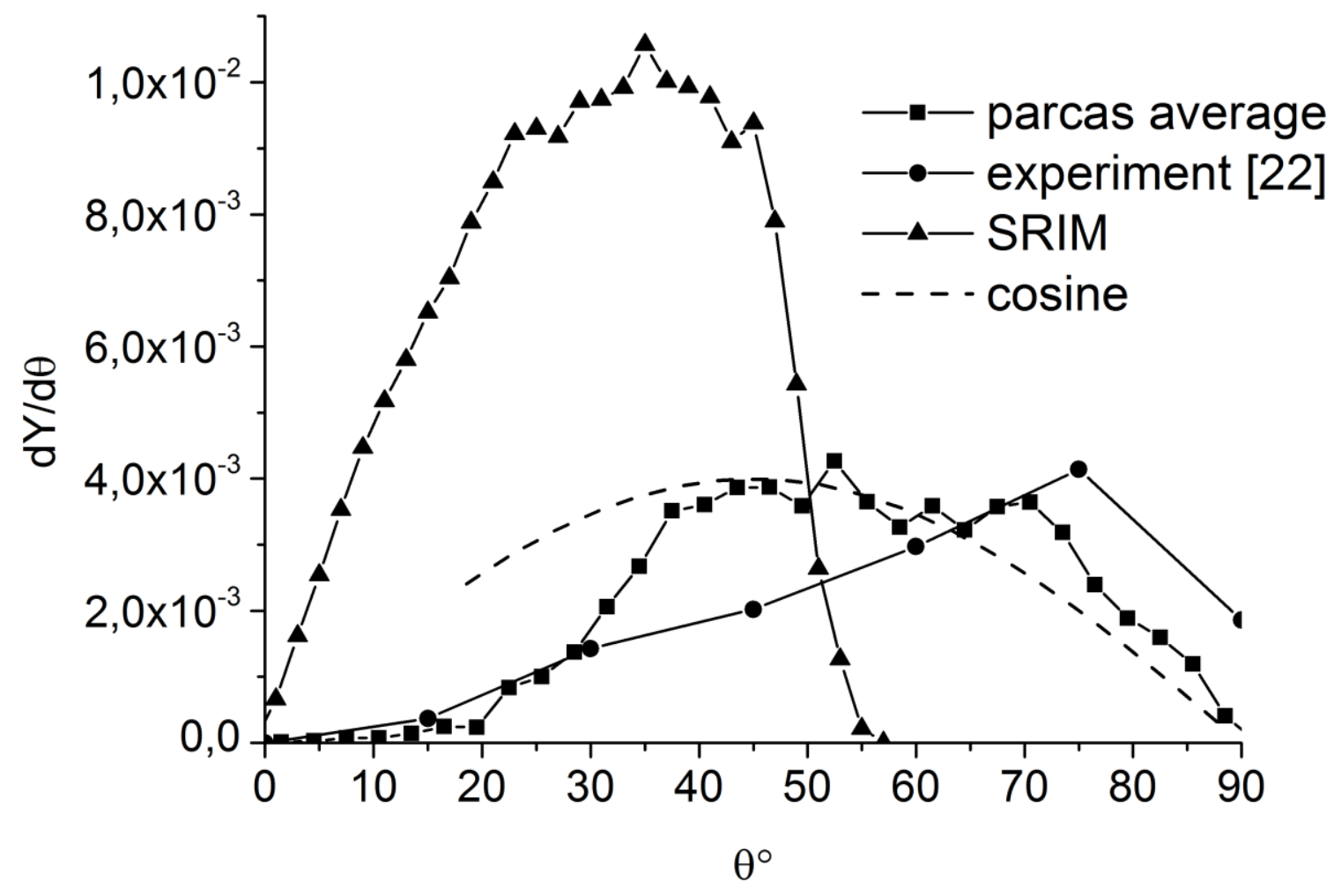

Fig. 5

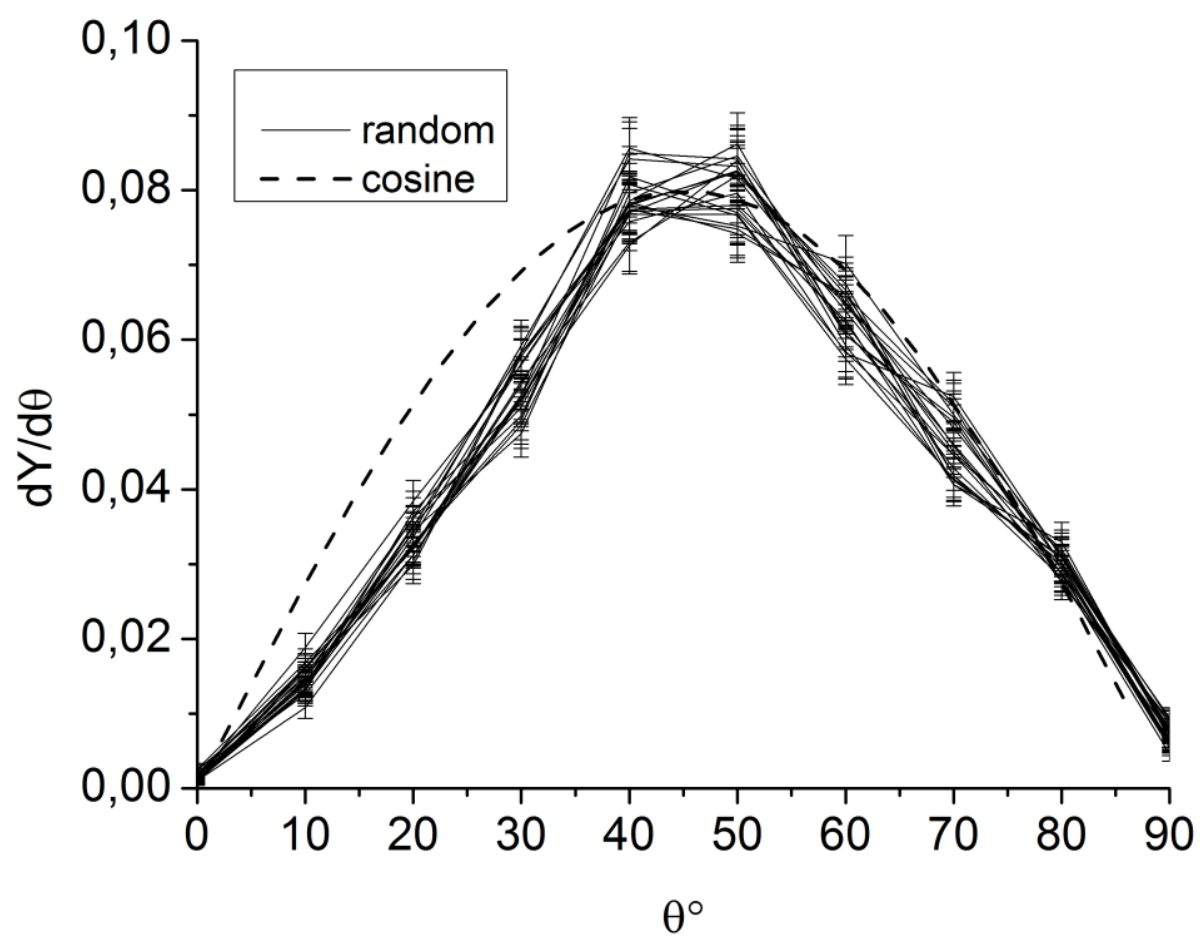

Fig. 6a 


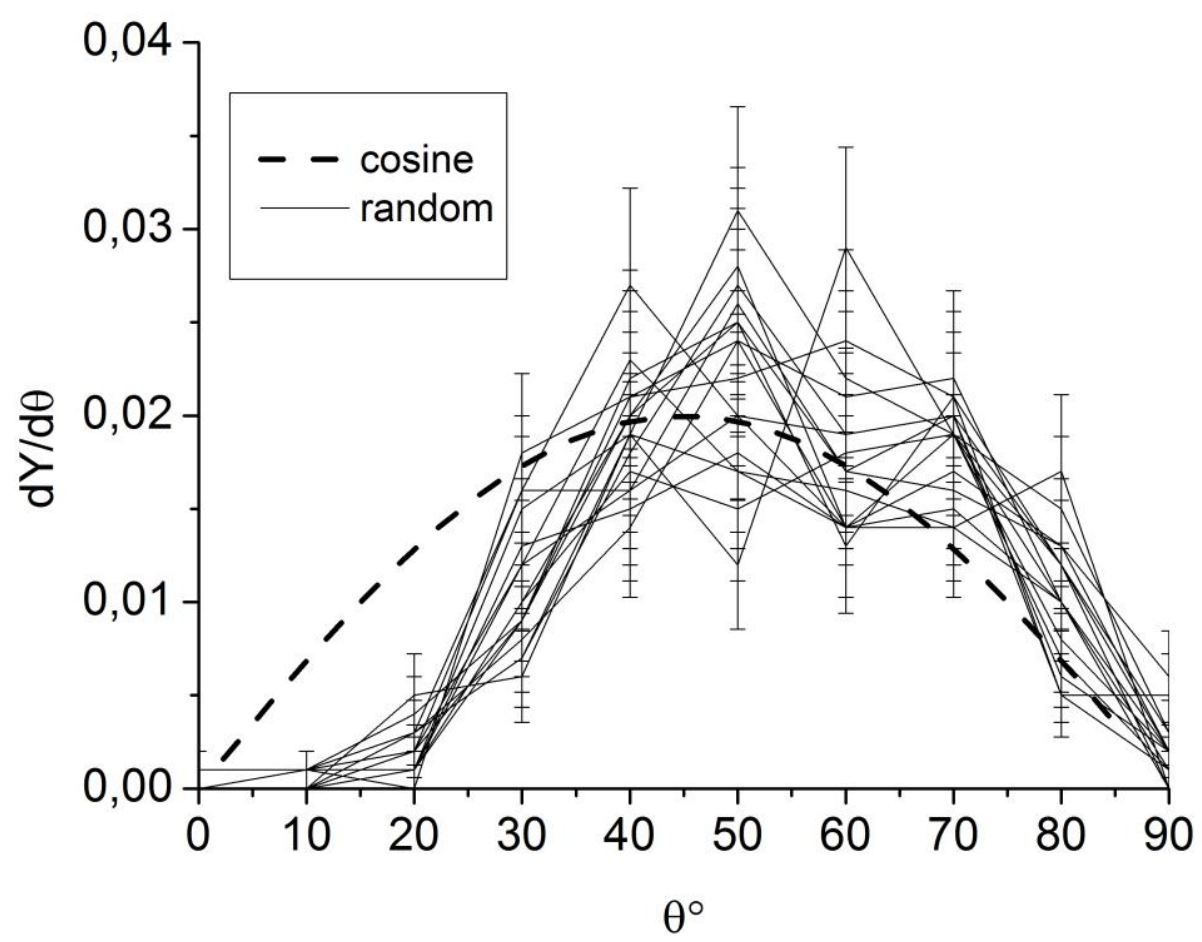

Fig. $6 b$
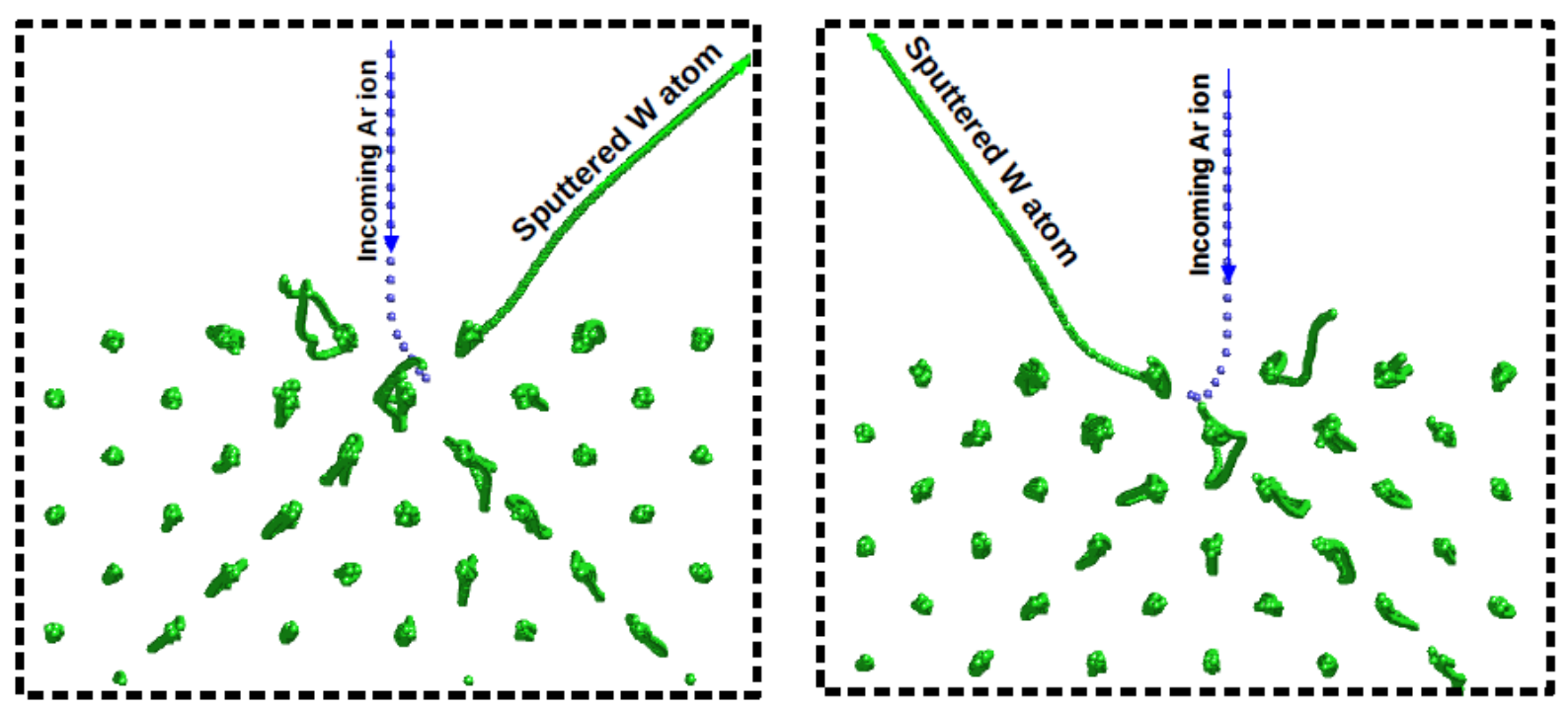

Fig. 7 


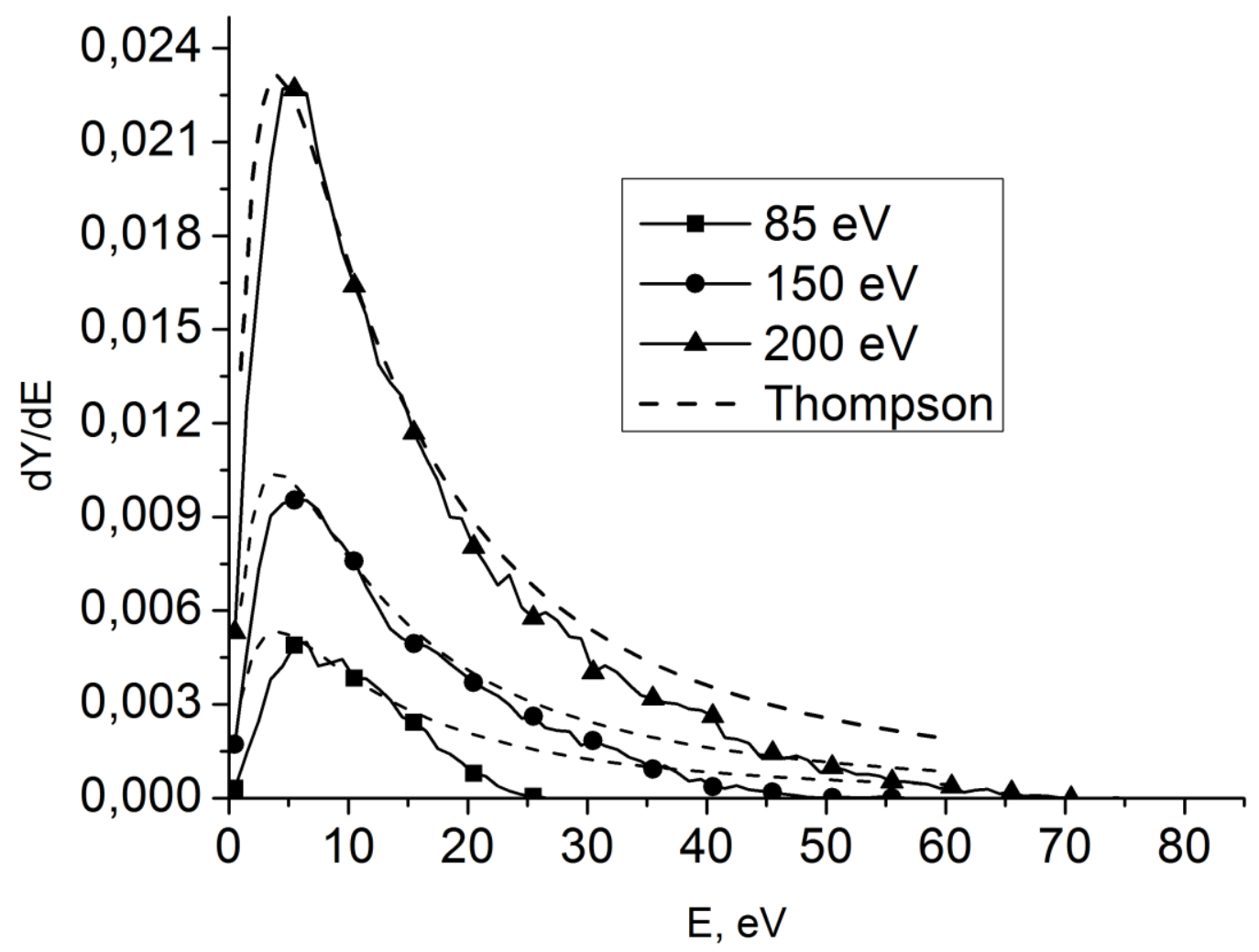

Fig. 8 